\title{
Valuation and hedging of weather derivatives on monthly average temperature
}

\author{
Yuji Yamada \\ Graduate School of Business Sciences, University of Tsukuba, 3-29-1 Otsuka, Bunkyo-ku, \\ Tokyo 112-0012, Japan; email: yuji@gssm.otsuka.tsukuba.ac.jp
}

\begin{abstract}
In this paper, we develop two types of pricing approach, one based on the utility indifference valuation and the other on non-parametric trend prediction, and estimate their hedge effect on energy businesses using empirical data. First, we consider an over-the-counter market for weather derivatives between an insurance company and an industry that runs a project affected by a weather index, for example, average temperature. We demonstrate supply and demand lines corresponding to those two positions, derive the equilibrium price and volume based on the exponential utility function and then generalize the result for a multi-period case so that swap contracts are carried out in continuous-time settings. Next, we introduce a trend-prediction-based pricing technique and analyze the hedge effect of weather derivatives on energy businesses. The historical simulation shows that the future contracts are highly effective for hedging electricity revenue when the revenue is proportional to the electricity sales in summer. Moreover, we demonstrate an optimal revenue structure with respect to the electricity sales when put options are used.
\end{abstract}

\section{INTRODUCTION}

Weather derivatives are contracts written on weather indices, which in turn are variables whose values are constructed from weather data (eg, Geman (1999)). In this paper, we consider the pricing of temperature-based weather derivatives and demonstrate their hedge effect on electric power businesses.

In the case of future contracts for a monthly average temperature, the investors have to determine a fixed value (or a future price) for trading the monthly average temperature in the future. If the actual monthly average temperature is higher than the fixed value (or the future price), the investor with the future long position can receive a payoff defined by their difference. If the actual temperature is lower than the future price, the buyer has to pay the difference to the seller. Although this type of future contract may be a simple example of weather derivatives, it is difficult to compute its future price theoretically owing to the incompleteness of the market (Davis (1998)), ie, there is no traded underlying asset for weather derivatives and, as a result, the replicating portfolio cannot be constructed, see

The author would like to thank H. Tsubaki from the University of Tsukuba, Japan and M. Iida from Mizuho Corporate Bank, Ltd, for their helpful comments and discussions. 
Cao and Wei (2004), Davis (2001) and Platen and West (2004) for pricing weather derivatives using incomplete market theory.

In this paper, we first provide a utility-based approach to find the future price of weather derivatives in an over-the-counter (OTC) market, where the contract is assumed to be carried out between an insurance company and an industry that runs a project affected by a weather index, for example, the average temperature. This situation is typical in the Japanese weather derivatives market, because most contracts are offered by insurance/finance companies and their price should be determined by taking asymmetric positions into account. Using an exponential utility function, we discuss the equilibrium price and volume of the future contracts. The result is also generalized to the multi-period case so that swap contracts are carried out in continuous-time settings. Next, we develop a pricing technique based on the trend prediction for temperature derivatives. We demonstrate the hedge effect of weather derivatives on the electricity revenue using future and put option contracts.

This paper is organized as follows. In Section 2, we describe weather derivatives on the monthly average temperature. Next, we discuss a utility-based approach for pricing weather derivatives in the OTC market in Section 3. In Section 4, we show a pricing technique based on trend prediction and provide some preliminary data analysis, where the relation between the power consumption and the temperature in Japan is demonstrated. The hedge effect of weather derivatives on energy businesses is analyzed in Section 5. The historical simulation shows that the weather future is highly effective for hedging electricity revenue when the revenue is proportional to the electricity sales in summer. Moreover, we compute an optimal revenue structure with respect to the electricity sales when put options are used in Section 6. Finally, Section 7 offers some concluding remarks.

\section{WEATHER DERIVATIVES ON THE MONTHLY AVERAGE TEMPERATURE}

Let $n(=0,1,2, \ldots)$ be a time step corresponding to the $n$th month from a certain initial month and let $T_{n}$ be the average temperature of that month. A future contract on the monthly average temperature is a derivative contract whose payoff at the end of the $n$th month is given by:

$$
\alpha\left(T_{n}-F_{n}\right)
$$

where $\alpha>0$ is some constant. Here $F_{n}$ is a future price (temperature) which is determined and is fixed when entering the future market (eg, at time zero) without any cost, ie, no initial payment is required for future contracts. Figure 1 explains an example of payoff structures for a future long position. If the actual average temperature of the $n$th month is higher than the future price, for example, $T_{n}=28^{\circ} \mathrm{C}$ and $F_{n}=25^{\circ} \mathrm{C}$, an investor with the future long position will receive $\mathrm{US} \$ 100 \times(28-25)=\mathrm{US} \$ 300$ when $\alpha=\mathrm{US} \$ 100$.

In the case of European-type options, the payoff may be given as:

$$
\alpha \max \left(T_{n}-K, 0\right)
$$


FIGURE 1 Payoff of a future contract.

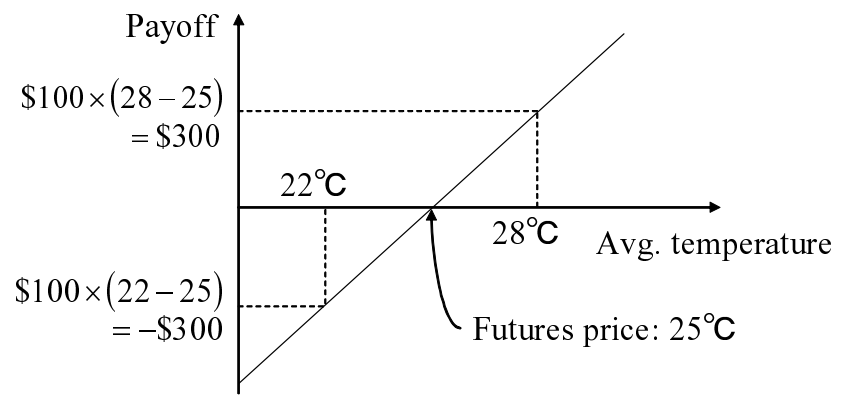

for a call and:

$$
\alpha \max \left(K-T_{n}, 0\right)
$$

for a put, where $K$ is a strike. One of the main differences of European-type call/put options from future contracts is that the payoff of a call/put is always positive and that it generally requires an initial payment or premium for the buyer.

In order to deal with the pricing problems in the same framework, here we consider future-type option contracts, where the contracts are carried out with no initial cost but instead the buyer has to make a (fixed) payment, $\hat{F}_{n}$, at the maturity $n$. In this case, the payoff of the call/put at the maturity will be given as:

$$
\alpha\left[\max \left(T_{n}-K, 0\right)-\hat{F}_{n}\right]
$$

or:

$$
\alpha\left[\max \left(K-T_{n}, 0\right)-\hat{F}_{n}\right]
$$

respectively. Here $\hat{F}_{n}$ is specified at the initial time (eg, time zero) when the contract is carried out and is considered as a future price of the option. If we discount $\hat{F}_{n}$ back to the initial time using a monthly risk-free interest rate $r$, then it is possible to compute the value of an option that pays (2) or (3) at the maturity $n$, ie, the value of the option at time $k(<n)$ is given as:

$$
\exp ^{-r(n-k)} \alpha \hat{F}_{n}
$$

For simplicity, we assume that $\alpha=1$.

\section{UTILITY-BASED VALUATION FOR THE FUTURE CONTRACT}

In this section, we discuss a utility-based valuation technique for the future contract in the OTC market. 


\subsection{Single-period case}

Consider a single-period case where the future contract is carried out at time zero for the $N$ th month's average temperature, $T_{N}$, and let $X_{N}:=T_{N}$ be the value of underlying. Assume that there are two investors that take different positions (long and short) in the future market of $X_{N}$. Note that those investors are willing to hedge their business portfolios by using the future contract, and let:

- $Y_{N}$ be the business profit at time $N$ for the investor with a future long position; and

- $Z_{N}$ be the business profit at time $N$ for the investor with a future short position.

We would like to derive a future price or price range that the two investors can agree to trade at.

For simplicity, suppose that the utility functions are exponential for both investors with different risk-aversion coefficients, $\alpha_{1}$ (future long position) and $\alpha_{\mathrm{s}}$ (future short position), ie:

$$
\begin{array}{cl}
U_{1}(w)=\exp \left(-\alpha_{1} w\right) & \text { (future long position) } \\
U_{\mathrm{s}}(w)=\exp \left(-\alpha_{\mathrm{s}} w\right) & \text { (future short position) }
\end{array}
$$

and that $X_{N}$ follows a Gaussian distribution with mean $\mu$ and variance $\sigma^{2}$, ie:

$$
X_{N} \sim \mathcal{N}\left(\mu, \sigma^{2}\right)
$$

Furthermore, we assume that the business profits of the two investors, $Y_{N}$ and $Z_{N}$, are Gaussian random variables satisfying:

$$
\begin{array}{ll}
Y_{N} \sim \mathcal{N}\left(\mu_{y}, \sigma_{y}^{2}\right), & \operatorname{Corr}\left(Y_{N}, X_{N}\right)=\rho_{x y} \\
Z_{N} \sim \mathcal{N}\left(\mu_{z}, \sigma_{z}^{2}\right), & \operatorname{Corr}\left(Z_{N}, X_{N}\right)=\rho_{x z}
\end{array}
$$

where $\operatorname{Corr}(\cdot, \cdot)$ stands for correlation of random variables.

With the above assumptions, we compute the utility indifference prices (see Cont and Tankov (2004) and Henderson and Hobson (2007) and references therein) corresponding to the future buying price and the future selling price. Let $F_{1}$ be a value that satisfies the following utility indifference condition for the future long position:

$$
\mathbb{E}\left[U\left(\delta_{1}\left(X_{N}-F_{1}\right)+Y_{N}\right)\right]=\mathbb{E}\left[U\left(Y_{N}\right)\right]
$$

where $\delta_{1}$ is the volume of the future (buying) contract. Note that $F_{1}$ can be thought of as the future (buying) price that the investor with the business profit $Y_{N}$ feels indifferent to with or without taking the future long position for $\delta$ units of future contracts. In this sense, $F_{1}$ provides an upper price bound for the future long position. On the other hand, the utility indifference future selling price, denoted by $F_{\mathrm{s}}$, is given to satisfy:

$$
\mathbb{E}\left[U\left(\delta_{\mathrm{S}}\left(F_{\mathrm{s}}-X_{N}\right)+Z_{N}\right)\right]=\mathbb{E}\left[U\left(Z_{N}\right)\right]
$$


FIGURE 2 Future selling price $F_{\mathrm{s}}$ versus volume $\delta_{\mathrm{s}}$.

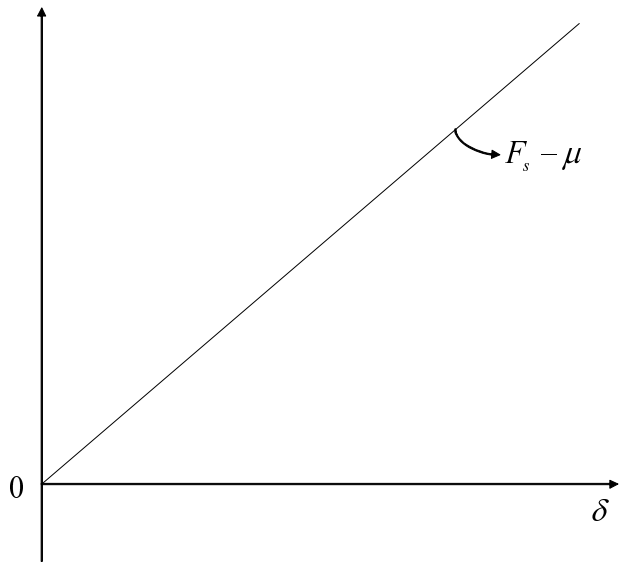

where $\delta_{1}$ is the volume of the future selling contract. In contrast with the long position's case, $F_{\mathrm{s}}$ may provide a lower price bound for the future short position. It is easy to show that the solutions of (10) and (11) are, respectively, given as:

$$
\begin{aligned}
& F_{1}=\mu-\frac{\alpha_{1} \sigma^{2}}{2} \delta_{1}-\alpha_{1} \sigma \sigma_{y} \rho_{x y} \\
& F_{\mathrm{s}}=\mu+\frac{\alpha_{\mathrm{s}} \sigma^{2}}{2} \delta_{\mathrm{s}}-\alpha_{\mathrm{s}} \sigma \sigma_{z} \rho_{x z}
\end{aligned}
$$

Now, consider the case that the future contract is carried out between an industry with $\rho_{x y}<0$ that takes a long position (buyer) and an insurance company with $\rho_{x z}=0$ that takes a short position (seller). Note that this situation is typical in the Japanese weather derivatives market where most contracts are sold by insurance/finance companies in the OTC market. In this case, the future selling price may be simplified to:

$$
F_{\mathrm{s}}=\mu+\frac{\alpha_{\mathrm{s}} \sigma^{2}}{2} \delta_{\mathrm{s}}
$$

Figure 2 shows the relation between the future selling price $F_{\mathrm{S}}$ and the volume $\delta_{\mathrm{s}}$ that may be offered by the insurance company. Note that the straight line in Figure 2 may be considered as a supply line from the insurance company in the sense that the insurance company is willing to sell the contract up to $\delta_{\mathrm{S}}$ with the future price of $F_{\mathrm{s}}$.

On the other hand, the demand line of the industry (buyer side) may be depicted as another straight line in Figure 3 . These two lines cross at point $(\bar{\delta}, \bar{F})$, 
FIGURE 3 The supply and demand lines for the future contract.

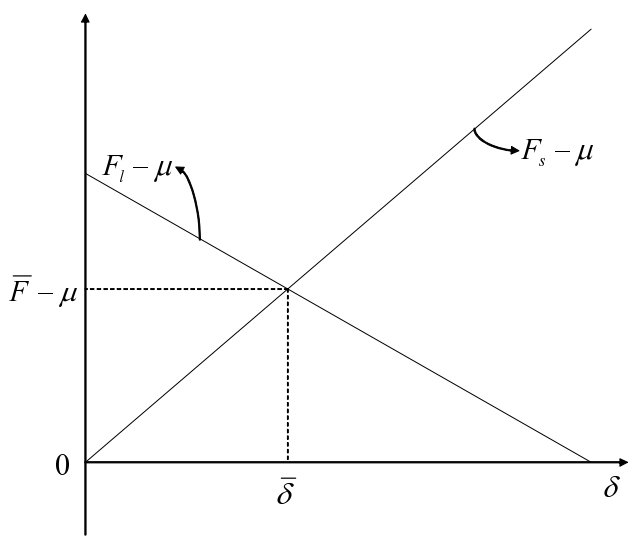

where:

$$
\begin{gathered}
\bar{\delta}=-\frac{2 \alpha_{1} \rho_{x y} \sigma_{y}}{\left(\alpha_{\mathrm{s}}+\alpha_{1}\right) \sigma} \\
\bar{F}=\mu-\frac{\alpha_{\mathrm{s}} \bar{\delta}}{2} \sigma^{2}=\mu-\frac{\alpha_{\mathrm{s}} \alpha_{1} \rho_{x y} \sigma_{y} \sigma}{\left(\alpha_{\mathrm{s}}+\alpha_{1}\right)}
\end{gathered}
$$

As the two investors would have dealings if $F_{\mathrm{s}} \leq F_{1}$ and would not have dealings when $F_{\mathrm{S}}>F_{1}$, the price satisfying $F_{1}=F_{\mathrm{S}}(=\bar{F})$ may be considered as an equilibrium price and $\delta(=\bar{\delta})$ such that $F_{1}=F_{\mathrm{S}}$ as the equilibrium volume.

Note that the above result may be generalized to the multi-agent case, and the problem can be formulated as a trade between the two representative investors for a set of sellers and a set of buyers, see Appendix A for further details. Also note that this equilibrium model is closely related to the so-called CARA-Normal model (eg, Li (2004) and references therein), and a comparison with CARANormal model is demonstrated in Appendix B.

\subsection{Multi-period case}

Next, we consider a continuous-time setting. Let $X(t)$ be the value of the underlying at time $t \in\left[t_{0}, t_{1}\right]$, which possibly represents the average temperature at day $t$ (or the $t$ th month as in the previous section). Consider a series of future contracts (or continuous swap) with different maturities $t \in\left[t_{0}, t_{1}\right]$, where each future contract has an instantaneous volume $\delta(t) \mathrm{d} t$. Let $F(t)$ be the future price with maturity $t \in\left[t_{0}, t_{1}\right]$. Then the instantaneous cashflow for a future long position (buyer) at time $t \in\left[t_{0}, t_{1}\right]$ is given as

$$
\delta(t)(X(t)-F(t)) \mathrm{d} t
$$


Then the utility indifference future price for the future long position, denoted by $F_{l}(t)$, may be given to satisfy the following condition:

$$
\begin{array}{rl}
\sup _{\phi \in \mathcal{S}} & \mathbb{E}\left[U\left(x+\int_{t_{0}}^{t_{1}} \delta(t)\left[X(t)-F_{l}(t)\right] \mathrm{d} t+\int_{t_{0}}^{t_{1}} \phi(t) \mathrm{d} S\right)\right] \\
= & \sup _{\phi \in \mathcal{S}} \mathbb{E}\left[U\left(x+\int_{t_{0}}^{t_{1}} \phi(t) \mathrm{d} S\right)\right]
\end{array}
$$

where $S(t)$ is the value of an asset (eg, stock) or a business portfolio, $\mathcal{S}$ is a set of self-financing strategies and $x$ is the initial wealth. Note that, in the case of a future short position (seller), $F_{1}(t)$ may be replaced by the future selling price, denoted by $F_{\mathrm{S}}(t)$, and the plus sign in front of $\delta$ becomes a minus.

Here we make several more assumptions:

1. the utility functions are exponential for both the seller (short position) and the buyer (long position), whose risk-aversion coefficients are, respectively, given as $\alpha_{\mathrm{s}}$ and $\alpha_{1}$;

2. $X(t) \sim \mathcal{N}\left(\mu(t), \sigma_{y}^{2}(t)\right)$; and

3. $\phi=0$ for the seller, where:

$$
\phi(t) \mathrm{d} S=Y(t) \mathrm{d} t
$$

for the buyer, where:

$$
Y(t) \sim \mathcal{N}\left(v(t), \sigma_{y}^{2}(t)\right)
$$

With these assumptions, the utility indifference future prices for the seller and the buyer, $F_{\mathrm{S}}(t)$ and $F_{1}(t)$, satisfy the following conditions, respectively:

$$
\begin{aligned}
\mathbb{E}\left[\exp \left(-\int_{t_{0}}^{t_{1}} \delta(t)\left[X(t)-F_{\mathrm{s}}(t)\right] \mathrm{d} t\right)\right] & =\exp (0)=1 \\
\mathbb{E}\left[\exp \left(\int_{t_{0}}^{t_{1}} \delta(t)\left[X(t)-F_{1}(t)\right] \mathrm{d} t+\int_{t_{0}}^{t_{1}} Y(t) \mathrm{d} t\right)\right] & =\mathbb{E}\left[\exp \left(\int_{t_{0}}^{t_{1}} Y(t) \mathrm{d} t\right)\right]
\end{aligned}
$$

By solving the above equations with respect to $F_{\mathrm{S}}(t)$ and $F_{1}(t)$, we obtain the following proposition.

PROPOSITION 1 The utility indifference future prices for the seller and the buyer, $F_{l}$ and $F_{s}$, satisfying (22) and (21) are given as

$$
\begin{aligned}
F_{l}(t)= & \mu(t)-\alpha_{1} \int_{t_{0}}^{t}\left\{\delta_{1}(v) \operatorname{Cov}(X(t), X(v))\right. \\
& \left.+\operatorname{Cov}(X(t), Y(v))+\frac{\delta_{1}(v)}{\delta_{1}(t)} \operatorname{Cov}(X(v), Y(t))\right\} \mathrm{d} v \\
F_{S}(t)= & \mu(t)+\alpha_{\mathrm{S}} \int_{t_{0}}^{t} \delta_{\mathrm{S}}(v) \operatorname{Cov}(X(t), X(v)) \mathrm{d} v
\end{aligned}
$$

where $\operatorname{Cov}(\cdot, \cdot)$ denotes a covariance of two random variables. 
Let $F_{1}(t)=F_{\mathrm{S}}(t)$ in Proposition 1. Then the following proposition holds for the volume function $\delta(t)$, which gives an equilibrium condition for the continuous case.

PROPOSITION 2 The equilibrium volume of $\delta(t)$ satisfies the following condition:

$$
\begin{aligned}
& \alpha_{\mathrm{s}} \int_{t_{0}}^{t} \delta(v) \operatorname{Cov}(X(t), X(v)) \mathrm{d} v \\
& =-\alpha_{1} \int_{t_{0}}^{t}\{\delta(v) \operatorname{Cov}(X(t), X(v))+\operatorname{Cov}(X(t), Y(v)) \\
& \left.\quad+\frac{\delta(v)}{\delta(t)} \operatorname{Cov}(X(v), Y(t))\right\} \mathrm{d} v \\
& \Leftrightarrow \int_{t_{0}}^{t}\left\{\left(\alpha_{\mathrm{s}}+\alpha_{1}\right) \delta(v) \operatorname{Cov}(X(t), X(v))+\alpha_{1} \operatorname{Cov}(X(t), Y(v))\right. \\
& \left.\quad+\alpha_{1} \frac{\delta(v)}{\delta(t)} \operatorname{Cov}(X(v), Y(t))\right\} \mathrm{d} v=0
\end{aligned}
$$

We provide a recursive algorithm to find the equilibrium volume of $\delta(t)$ satisfying (25) as follows.

Step 1. Discretize Equation (25) as:

$$
\sum_{i=1}^{n}\left[\left(\alpha_{\mathrm{s}}+\alpha_{1}\right) \delta_{n} \delta_{i} \operatorname{Cov}\left(X_{i}, X_{n}\right)+\alpha_{1} \delta_{n} \operatorname{Cov}\left(X_{n}, Y_{i}\right)+\alpha_{1} \delta_{i} \operatorname{Cov}\left(X_{i}, Y_{n}\right)\right]=0
$$

Step 2. Solve Equation (26) with respect to $n=1, \ldots, N$, recursively, as follows.

- Case $n=1$ :

$$
\begin{gathered}
\left(\alpha_{\mathrm{S}}+\alpha_{1}\right) \delta_{1} \operatorname{Cov}\left(X_{1}, X_{1}\right)+\alpha_{1} \operatorname{Cov}\left(X_{1}, Y_{1}\right)+\alpha_{1} \operatorname{Cov}\left(X_{1}, Y_{1}\right)=0 \\
\Leftrightarrow \delta_{1}=-\frac{2 \alpha_{1} \operatorname{Cov}\left(X_{1}, Y_{1}\right)}{\left(\alpha_{\mathrm{S}}+\alpha_{1}\right) \sigma_{1}^{2}}
\end{gathered}
$$

- Case $n \geq 2$ :

$$
\begin{aligned}
& \left(\alpha_{\mathrm{s}}+\alpha_{1}\right) \sigma_{n}^{2} \delta_{n}^{2} \\
& +\left[2 \alpha_{1} \operatorname{Cov}\left(X_{n}, Y_{n}\right)+\sum_{i=1}^{n-1}\left\{\left(\alpha_{\mathrm{s}}+\alpha_{1}\right) \delta_{i} \operatorname{Cov}\left(X_{i}, X_{n}\right)\right.\right. \\
& \left.\left.\quad+\alpha_{1} \operatorname{Cov}\left(X_{n}, Y_{i}\right)\right\}\right] \delta_{n}+\sum_{i=1}^{n-1} \alpha_{1} \delta_{i} \operatorname{Cov}\left(X_{i}, Y_{n}\right)=0
\end{aligned}
$$




$$
\begin{aligned}
\Rightarrow & \delta_{n} \\
= & -\frac{2 \alpha_{1} \operatorname{Cov}\left(X_{n}, Y_{n}\right)+\sum_{i=1}^{n-1}\left\{\left(\alpha_{\mathrm{s}}+\alpha_{1}\right) \delta_{i} \operatorname{Cov}\left(X_{i}, X_{n}\right)+\alpha_{1} \operatorname{Cov}\left(X_{n}, Y_{i}\right)\right\}}{2\left(\alpha_{\mathrm{s}}+\alpha_{1}\right) \sigma_{n}^{2}} \\
& +\frac{1}{2\left(\alpha_{\mathrm{s}}+\alpha_{1}\right) \sigma_{n}^{2}}\left[\left\{2 \alpha_{1} \operatorname{Cov}\left(X_{n}, Y_{n}\right)+\sum_{i=1}^{n-1}\left\{\left(\alpha_{\mathrm{s}}+\alpha_{1}\right) \delta_{i} \operatorname{Cov}\left(X_{i}, X_{n}\right)\right.\right.\right. \\
& \left.\left.\left.+\alpha_{1} \operatorname{Cov}\left(X_{n}, Y_{i}\right)\right\}\right\}^{2}-4\left(\alpha_{\mathrm{s}}+\alpha_{1}\right) \sigma_{n}^{2}\left\{\sum_{i=1}^{n-1} \alpha_{1} \delta_{i} \operatorname{Cov}\left(X_{i}, Y_{n}\right)\right\}\right]^{\frac{1}{2}}
\end{aligned}
$$

\section{PRICING BASED ON TREND PREDICTION AND PRELIMINARY DATA ANALYSIS}

The second half of this paper is devoted to developing another pricing technique based on trend prediction. As this methodology is closely related to statistical analysis for empirical data, we also demonstrate an empirical analysis for pricing weather derivatives based on the trend prediction and estimate its hedge effect on the power energy businesses.

\subsection{Pricing based on trend prediction}

Let $T_{n}, n=0,1,2, \ldots$, be the monthly average temperature of the $n$th month. The pricing technique based on the trend prediction consists of the following two steps.

1. Assume that $C_{n}$ is given by one of the following values:

$$
C_{n}= \begin{cases}T_{n} & \text { (future contract) } \\ \max \left(T_{n}-K, 0\right) & \text { (call option) } \\ \max \left(K-T_{n}, 0\right) & \text { (put option) }\end{cases}
$$

2. Express $C_{n}$ as:

$$
C_{n}=f(n)+\epsilon_{n}, \quad \operatorname{Mean}\left(\epsilon_{n}\right)=0
$$

using a function $f(\cdot)$ and residuals $\epsilon_{n}$, where Mean $(\cdot)$ is a sample mean.

Here the function $f(\cdot)$ may be determined, eg, using a parametric method such as polynomial regression if sample data is given, by minimizing the sample variance of $\epsilon_{n}$ under the zero sample mean constraint. Instead of solving polynomial regression, we can solve a non-parametric regression problem to find a (cubic) smoothing spline that minimizes the following generalized sum of squares (PRSS; eg, Hastie and Tibshirani (1990)),

$$
\operatorname{PRSS}=\sum_{i=1}^{N}\left\{C_{n}-f(n)\right\}^{2}+\lambda \int\left\{f^{\prime \prime}(n)\right\}^{2} \mathrm{~d} x
$$


where the PRSS is minimized among all functions $f(\cdot)$ with two continuous derivatives. In (32), the first term measures closeness to the data while the second term penalizes curvature in the function. Note that, if $\lambda=0$ and $f(\cdot)$ is given by a polynomial function, the problem reduces to the standard regression polynomial and is solved by the least squares method. It is shown that (32) has an explicit, unique minimizer and that regression splines can be extended to the multivariable case with additive sums of smoothing splines, known as "generalized additive models" (GAM; Hastie and Tibshirani (1990)). These can be computed using a free software R (see http://cran.r-project.org/) and we refer to the class of smoothing splines for non-parametric regression as GAM in this paper.

For the trend-prediction-based pricing technique, we set $F_{n}$ as $f(n)$ for future contracts or $\hat{F}_{n}$ as $f(n)$ for put options. In this case, the payoff of the future contract or the future type put option at time $n$ is given by $\epsilon_{n}$. As $\epsilon_{n}$ satisfies the zero mean constraint, Mean $\left(\epsilon_{n}\right)=0$, the expected value of the payoff is also zero and $F_{n}$ (or $\hat{F}_{n}$ ) gives the expectation of $C_{n}$. Note that, if we need to estimate the prices in the future period, we can use extrapolation technique based on regression polynomials or GAM.

In the following sections, we apply the trend-prediction-based pricing technique to analyze the hedge effect of weather derivatives on electricity revenue in the Japanese market. Before demonstrating our simulation results, we explain the long-term trend in power demand and discuss the relationship between electricity consumption and the temperature in Japan.

\subsection{Preliminary data analysis}

In our empirical analysis, we use the electricity consumption data of the Tokyo Electricity Power Company (TEPCO) for the period of April 1963 to December 2003, and the temperature data in Tokyo for the same period. ${ }^{1}$ Note that TEPCO covers not only Tokyo but also some other areas around Tokyo and that it actually carried out a weather derivative contract with the Tokyo Gas Supply Company (TGSC) based on daily average temperatures in summer, 2001 (see Kariya (2003) for details).

Let $P_{n}$ denote the electricity sales of the $n$th month in the data period and let $T_{n}$ be the monthly average temperature. Figure 4 shows the relationship between the excess electricity consumption and the average temperature for each month. The solid curve is a regression spline function which approximates the sample data based on GAM, where the excess electricity consumption is calculated by deducting the long-term trend (or the average electricity consumption of the year) from the monthly electricity sales. The dashed curves denote the $95 \%$ confidence interval.

\footnotetext{
${ }^{1}$ All of the electric power sales data in this paper can be downloaded from http://www3.fepc.or.jp/tokei/ (in Japanese) and the weather data including temperature can be obtained from Japan Meteorological Business Support Center (JMBSC) at http://www.jmbsc.or.jp/ (in Japanese).
} 
FIGURE 4 Electricity demand versus average temperature.

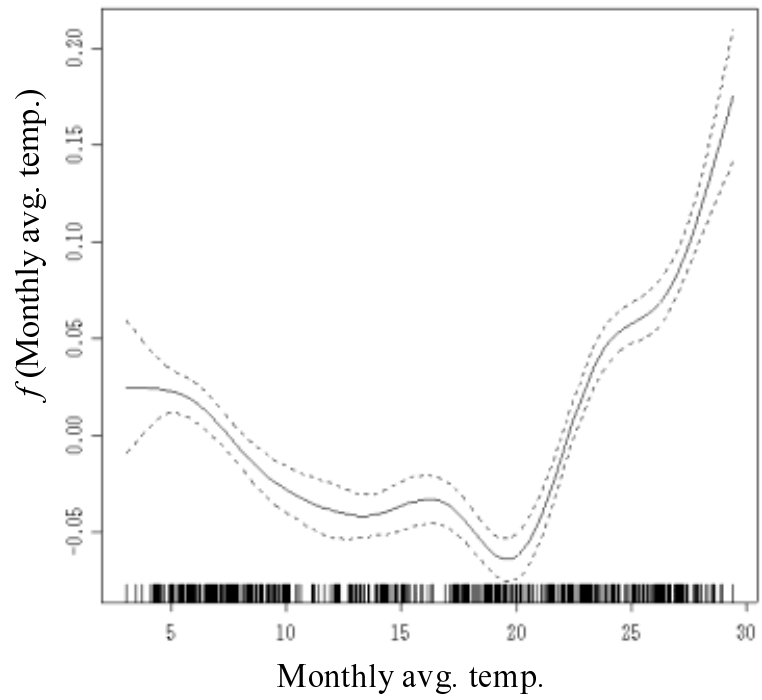

Figure 4 clearly indicates that the excess electricity consumption increases with the average temperature when the average temperature is greater than $20^{\circ} \mathrm{C}$ in summer. Even though a similar tendency is observed when the temperature decreases in winter, the sensitivity of electricity consumption with respect to changes in temperature seems much higher in summer than winter.

To observe tendencies in electricity sales in summer, we examined the data for June, July and August in the period of 1963-2003, and obtained Figure 5, where the electricity consumption is plotted for each month. Note that the solid curve is a third-order polynomial obtained from the least-squares approximation. We see that electricity consumption becomes volatile after the first oil crisis happened in 1973.

The argument in this section can be summarized as follows:

- the sensitivity of electricity consumption with respect to changes in temperature is higher in summer; and

- the electricity sales become volatile in the latter half of the 1970's, after the first oil crisis.

\section{ESTIMATION OF HEDGE EFFECT OF WEATHER DERIVATIVES}

In this section, we analyze the data in summer from the latter half of the 1970s to 2003 , where the sensitivity and the volatility of electricity consumption with respect to temperature are both higher; after this we demonstrate the hedge effect of weather derivatives on the electricity revenue. 
FIGURE 5 Changes of electricity sales in summer (Tokyo).

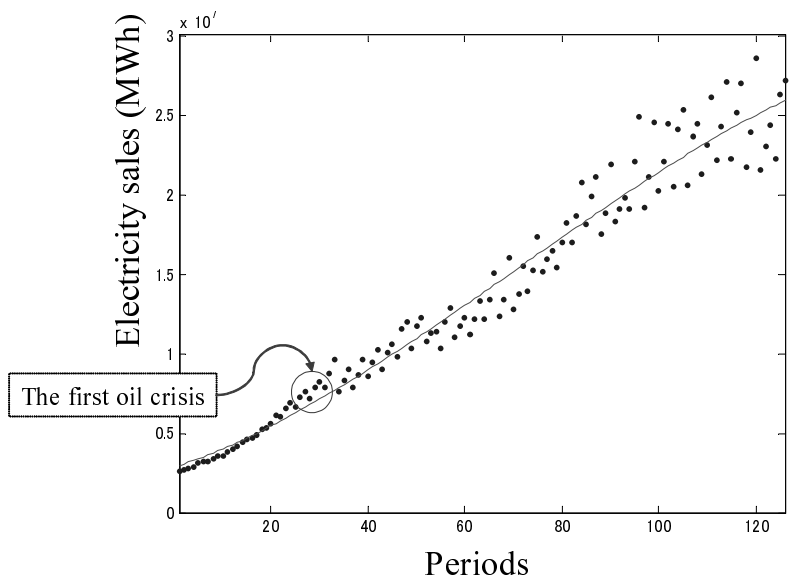

\subsection{Regression polynomials for temperature and electricity sales}

The fluctuations of monthly average temperatures for June, July and August from 1976 to 2003 is expressed in Figure 6, where the solid curve is computed using a third-order regression polynomial. If this line is obtained from the least-squares method with a zero mean constraint of the residuals, the trend can represent a future price. Also the difference between the average temperature and the trend provides the payoff for the future long position.

The change in electricity consumption for each month in the same period is shown in Figure 7, where the solid curve is a third-order regression polynomial that approximates the sample points in the least-squares sense. We make the following assumption.

ASSUMPTION 1 The revenue of the electricity power company, denoted by $R_{n}$, is proportional to the electricity sales, ie:

$$
R_{n}=c P_{n}
$$

where $R_{n}$ is the electricity revenue at each month.

As the electricity sector in Japan has not been liberalized completely yet, this assumption is not far from the real situation. It will turn out, under this assumption, that the use of futures contract provides the best hedge performance of all the derivatives on the average temperature using a minimum variance hedge. Let $\hat{R}_{n}$ be the revenue that deducted the trend, ie:

$$
\hat{R}_{n}=R_{n}-\text { Trend }_{n}
$$

Also let $\hat{Q}_{n}$ be the payoff of a weather derivative that can be used to hedge the electricity revenue. 
FIGURE 6 Monthly average temperature in summer 1976-2003 (Tokyo).

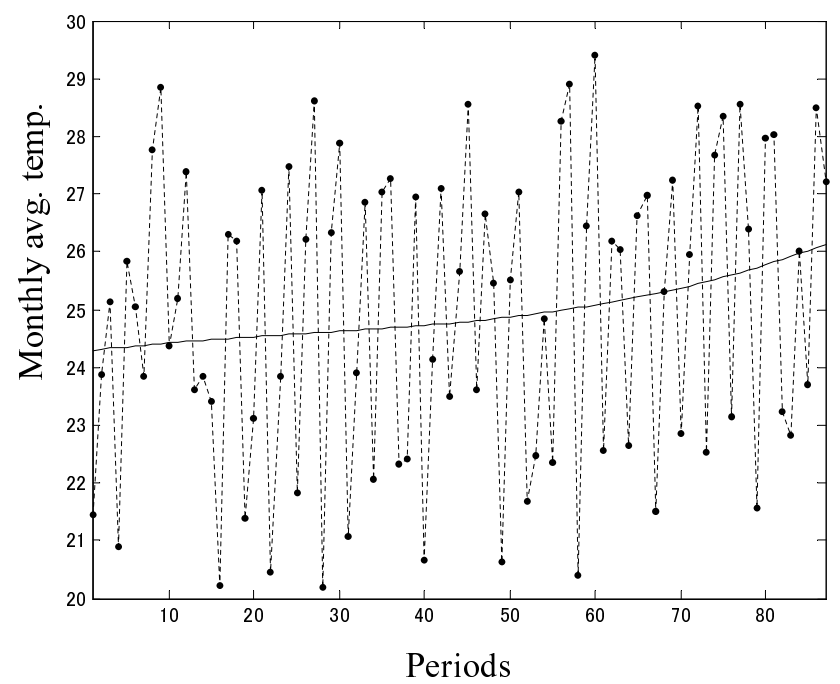

FIGURE 7 Electricity sales in summer 1976-2003 (Tokyo).

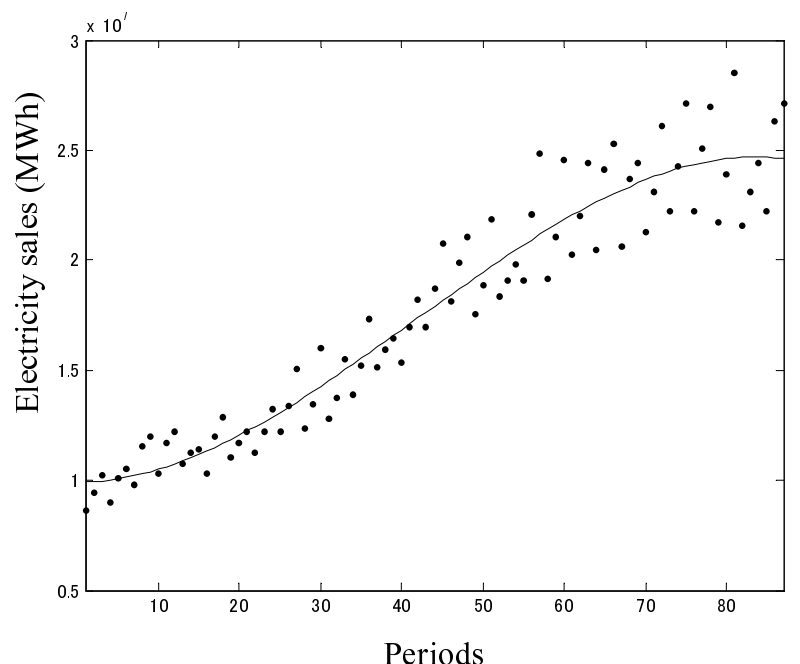

\subsection{Estimation of the hedge effect}

Now, we consider the following minimum variance hedge:

$$
\begin{array}{ll}
\text { Minimize } & \operatorname{Var}\left(\hat{R}_{n}+\Delta_{n} \hat{Q}_{n}\right) \\
\text { Subject to } & \text { mean }\left(\hat{Q}_{n}\right)=0
\end{array}
$$


where $\operatorname{Var}(\cdot)$ is a sample variance. By assuming that $\Delta_{n}$ is constant for all periods, the minimizer of $\Delta$ (denoted by $\left.\Delta^{*}\right)$ is given by:

$$
\Delta^{*}=-\frac{\sigma_{\mathrm{r}}}{\sigma_{z}} \rho_{q r}
$$

where $\sigma_{z}$ and $\sigma_{\text {r }}$ are the standard deviations of $\hat{Q}_{n}$ and $\hat{R}_{n}$, respectively, and $\rho_{q r}$ is the correlation coefficient between $\hat{R}_{n}$ and $\hat{Q}_{n}$. If $\rho_{q r}>0$, then it holds that $\Delta<0$ from (35). Therefore, we see that the electricity power company would like to take the short position of the futures contract, given that $\hat{R}_{n}$ and $\hat{Q}_{n}$ have a positive correlation. The minimum variance is then given as:

$$
\operatorname{Var}\left(P_{n}+\Delta^{*} \hat{Q}_{n}\right)=\sigma_{\mathrm{r}}^{2}\left(1-\rho_{q r}^{2}\right)
$$

If we divide the minimum variance by the variance of the original electricity revenue, then we have the "variance reduction rate":

$$
V_{\mathrm{r}}=\frac{\operatorname{Var}\left(P_{n}+\Delta^{*} \hat{Q}_{n}\right)}{\sigma_{\mathrm{r}}^{2}}=\frac{\sigma_{\mathrm{r}}^{2}\left(1-\rho_{q r}^{2}\right)}{\sigma_{\mathrm{r}}^{2}}=1-\rho_{q r}^{2}
$$

Here $V_{\mathrm{r}}$ satisfies:

$$
0 \leq V_{\mathrm{r}} \leq 1
$$

As a smaller $V_{\text {r }}$ provides a better variance reduction effect, we can conclude that a larger $\rho_{q r}^{2}$ leads to a smaller variance of the hedged electricity revenue. Note that the variance reduction rate is obtained once $\rho_{q r}$ is computed.

We calculate the correlation for the data sets in Figures 6 and 7. Figure 8 shows the relation between $\hat{R}_{n}$ and $\hat{Q}_{n}$ in this case and $\rho_{q r}$ is computed as:

$$
\rho_{q r}=0.811
$$

We see that $\hat{R}_{n}$ and $\hat{Q}_{n}$ are highly correlated. From (37), the variance reduction rate $V_{\mathrm{r}}$ may be obtained as:

$$
V_{\mathrm{r}}=0.342
$$

The same technique may be applied for a different data set in a different area, eg, Osaka. Figure 9 provides our simulation results, where the correlation between $\hat{R}_{n}$ and $\hat{Q}_{n}$ is computed for Kansai Electric Power Company and the temperature in Osaka for each month during the period 1976-2003. In this case, the correlation coefficient is given as:

$$
\rho_{q r}=0.875
$$

and the variance reduction rate $V_{\mathrm{r}}$ is given as:

$$
V_{\mathrm{r}}=0.235
$$

We see that the variance reduction rate is $10 \%$ lower than that of Tokyo.

Finally, we summarize the correlation coefficients between $\hat{R}_{n}$ and $\hat{Q}_{n}$ and the variance reduction rate for the three Japanese metropolitan cities: Tokyo, Osaka and Nagoya (see Table 1). Although these values vary depending on the area, on average we conclude that futures contracts on the average temperature are effective for hedging the electricity revenue if the electricity revenue is proportional to the sales. 
FIGURE 8 Average temperature versus electricity sales (Tokyo).

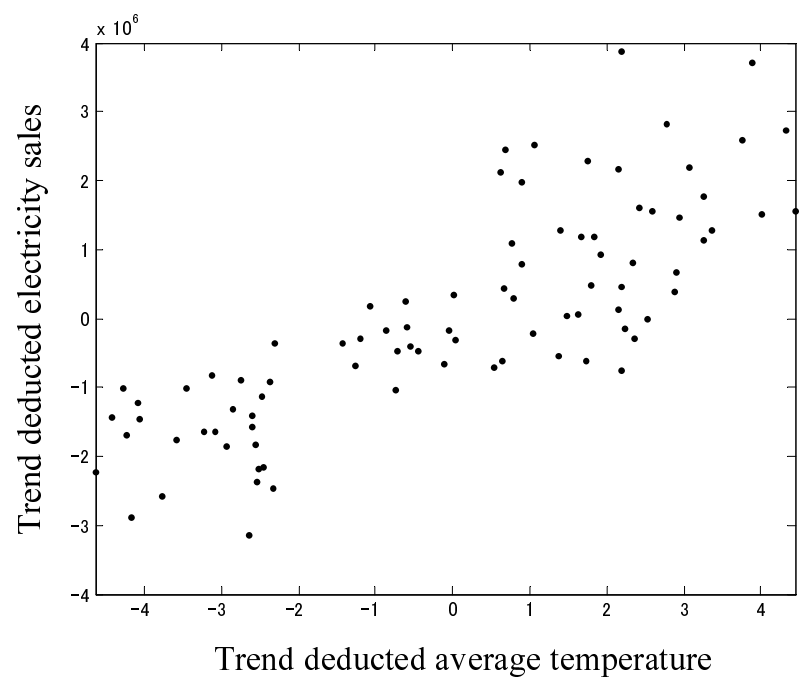

FIGURE 9 Average temperature versus electricity sales (Osaka).

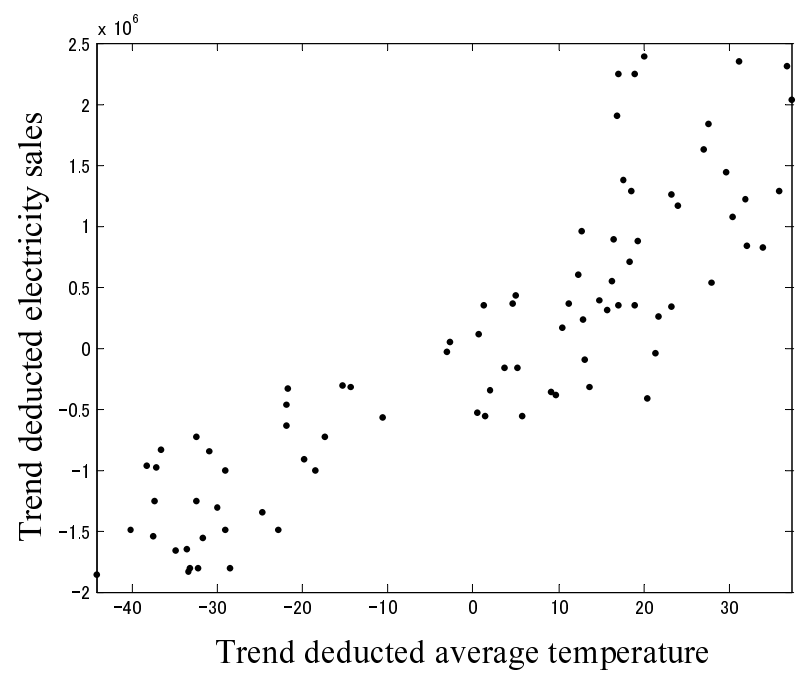

TABLE 1 Variance reduction rates for Tokyo, Osaka and Nagoya.

\begin{tabular}{lcc}
\hline City (power company) & $\boldsymbol{\rho}_{\boldsymbol{q} \boldsymbol{r}}$ & $\boldsymbol{V}_{\mathbf{r}}$ \\
\hline Tokyo (TEPCO) & 0.811 & 0.342 \\
Osaka (Kansai) & 0.875 & 0.235 \\
Nagoya (Chubu) & 0.847 & 0.283 \\
\hline
\end{tabular}




\section{HEDGE EFFECT USING GAMS AND THE CONSTRUCTION OF PUT OPTIONS}

In the previous section, we demonstrated the effectiveness of futures on the average temperature using polynomial regressions. Here we consider regression splines based on GAM to perform the same analysis. Furthermore, we illustrate the use of put options for hedging the electricity revenue efficiently.

\subsection{Estimation of hedge effect using GAM}

Let $\hat{R}_{n}$ be the revenue minus the trend and consider a smoothing spline function $f(\cdot)$ which satisfies the following relation:

$$
\hat{R}_{n}=f\left[\hat{Q}_{n}\right]+\eta_{n}, \quad \operatorname{Mean}\left(\eta_{n}\right)=0
$$

where $\hat{Q}_{n}$ and $\eta_{n}$ are, respectively, the trend deducted average temperature and the residuals. Assume that the PRSS of (43) is minimized by $f=f^{*}$ using GAMS and rewrite Equation (43) as:

$$
\eta_{n}=\hat{R}_{n}-f^{*}\left[\hat{Q}_{n}\right]
$$

If $f^{*}$ is a linear function, the resulting formula is the same as the minimum variance hedge given in Section 5, and the minimizer $\Delta^{*}$ may be obtained. In this sense, the minimum variance hedge is a special case of (44).

With Assumption 1 (ie, the revenue is proportional to the sales), we compute $f^{*}$ using GAM. Figure 10 shows our simulation result, where the function $f^{*}$ is expressed as the solid line. We can clearly see that the function $f^{*}$ is linear, which indicates that GAM provides the same result as the standard minimum variance hedge. This fact suggests that, if the profit of the electricity company is proportional to the sales, the use of futures provides the best hedge performance of all the derivatives on the average temperature. Note that, in this case, the variance reduction rate is computed as:

$$
V_{\mathrm{r}}=0.341
$$

which is almost the same as that obtained from the standard minimum variance hedge.

\subsection{Optimal revenue structure using put options}

Next, we consider the case where put options are used to hedge the electricity revenue. Consider a future-type put option as explained in Section 2. Suppose that the strike prices of the put options are given by the future prices of the underlying average temperature and that the future prices of the put options are computed based on GAM. Let $\hat{Q}_{n}$ be the payoff of holding such options. We are interested in the structure of the electricity revenue so that the use of the put options is effective. Recall that we assumed that the electricity revenue is proportional to the sales in Assumption 1. In this section, we consider the case where the excess revenue (denoted by $\hat{R}_{n}$ ) is a function of the trend deducted electricity sales $\hat{P}(n)$, 
FIGURE 10 Regression splines for average temperature versus electricity sales (Tokyo).

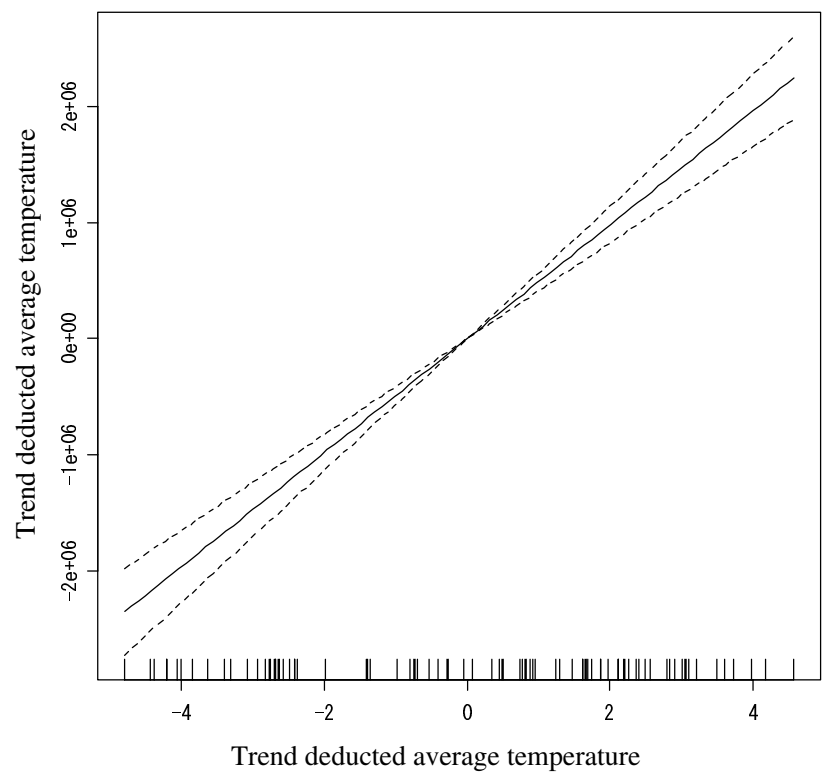

ie:

$$
\hat{R}=g[\hat{P}(n)]
$$

We compute $g=g^{*}$ to solve the following problem:

$$
\begin{array}{ll}
\text { Minimize } & \operatorname{PRSS}\left(g[\hat{P}(n)]+\hat{Q}_{n}\right) \\
\text { Subject to } & \operatorname{mean}\left(\hat{Q}_{n}\right)=0
\end{array}
$$

Note that the PRSS is defined as in (32) and $g^{*}$ may be found using GAM. The solution to the above problem gives the revenue size that can be hedged using one unit of a put option. If the electricity company were to hedge all of the revenue by put options, then the volume of the put options can be adjusted by scaling the function $g$ using some constant $k>0$ so that it matches the revenue size. Consequently, one can find the optimal revenue structure and the volume of put options for given electricity sales by applying GAM.

We solved the minimization problem in Equation (47) by constructing put options for the data sets of the average temperature and the electricity sales in Osaka, and obtained Figure 11. In this case, the variance reduction rate is computed as:

$$
V_{\mathrm{r}}=0.169
$$

which is much smaller than those in Table 1. From this simulation, we see that if the relation between the electricity revenue and the electricity sales is given by 
FIGURE 11 Optimal structure of electricity revenue for put options (Osaka).

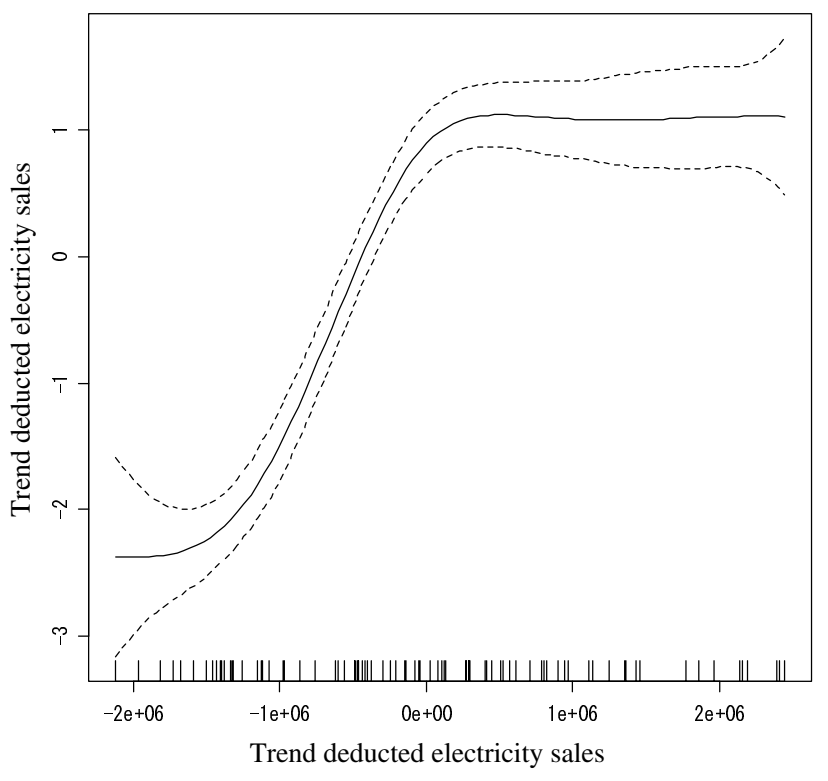

or is close to that expressed by $g^{*}(\cdot) \times$ constant, the electricity revenue can be hedged effectively using put options.

\section{CONCLUSION}

In this paper, we have introduced weather derivatives based on monthly average temperatures and developed two pricing techniques based on the utility indifference and trend predictions, respectively. Then, we discussed the hedge effect of the electricity business profit using weather derivatives. We have also demonstrated the effectiveness of weather derivatives on the Japanese electricity business. Our numerical simulations showed that futures on the average temperatures are effective for hedging the electricity revenue if the electricity revenue is proportional to the sales. Finally, we investigated the revenue structure that can be hedged effectively by put options.

\section{APPENDIX A MULTI-AGENT CASE}

Assume that there are $M$ seller agents, $1, \ldots, M$, whose risk-aversion coefficients are given as $\alpha_{s, 1}, \ldots, \alpha_{s, M}$, and let $\rho_{x z}=0$ for simplicity. In this case, their 
supply functions are given as follows:

$$
\left\{\begin{array} { c } 
{ F _ { \mathrm { S } } - \mu = \frac { \alpha _ { s , 1 } \sigma ^ { 2 } } { 2 } \delta _ { 1 } } \\
{ F _ { \mathrm { S } } - \mu = \frac { \alpha _ { s , 2 } \sigma ^ { 2 } } { 2 } \delta _ { 2 } } \\
{ \vdots } \\
{ F _ { \mathrm { S } } - \mu = \frac { \alpha _ { s , M } \sigma ^ { 2 } } { 2 } \delta _ { M } }
\end{array} \Leftrightarrow \left\{\begin{array}{c}
\frac{F_{\mathrm{S}}-\mu}{\alpha_{s, 1}}=\frac{\sigma^{2}}{2} \delta_{1} \\
\frac{F_{\mathrm{S}}-\mu}{\alpha_{s, 2}}=\frac{\sigma^{2}}{2} \delta_{2} \\
\vdots \\
\frac{F_{\mathrm{S}}-\mu}{\alpha_{s, M}}=\frac{\sigma^{2}}{2} \delta_{M}
\end{array}\right.\right.
$$

By taking the sum of both sides of the $M$ equations in (A.1), one can compute the aggregate demand function as:

$$
\left(F_{\mathrm{S}}-\mu\right)\left(\sum_{i=1}^{M} \frac{1}{\alpha_{s, i}}\right)=\frac{\sigma^{2}}{2}\left(\sum_{i=1}^{M} \delta_{i}\right)
$$

or, equivalently:

$$
\begin{gathered}
F_{\mathrm{s}}-\mu=\frac{\bar{\alpha}_{\mathrm{s}} \sigma^{2}}{2} \bar{\delta}_{\mathrm{s}} \\
\bar{\alpha}_{\mathrm{s}}:=\frac{1}{\sum_{i=1}^{M}\left(1 / \alpha_{s, i}\right)}, \quad \bar{\delta}_{\mathrm{s}}:=\sum_{i=1}^{M} \delta_{i}
\end{gathered}
$$

Similarly, the demand function for a buyer agent $i$ satisfies:

$$
\frac{F_{1}-\mu}{\alpha_{l, i}}=-\frac{\sigma^{2}}{2} \delta_{i}-\operatorname{Cov}\left(X_{N}, Y_{N}^{(i)}\right)
$$

where $\alpha_{1, i}$ is the risk-aversion coefficient for the agent $i$ and $Y_{N}^{(i)}$ is the profit from business portfolio for the agent $i$.

In this case, their aggregate demand function may be computed as follows:

$$
F_{1}-\mu=-\frac{\bar{\alpha}_{1} \sigma^{2}}{2} \bar{\delta}_{1}-\bar{\alpha}_{1} \operatorname{Cov}\left(X_{N}, \bar{Y}_{N}\right)
$$

where:

$$
\bar{\alpha}_{1}=\frac{1}{\sum_{i=1}^{M}\left(1 / \alpha_{1, i}\right)}, \quad \bar{\delta}_{1}=\sum_{i=1}^{M} \delta_{i}, \quad \hat{Y}_{N}=\sum_{i=1}^{M} Y_{N}^{(i)}
$$

If $\rho_{x z} \neq 0$, the aggregate demand function for the seller agents is given as:

$$
\begin{gathered}
F_{\mathrm{S}}-\mu=\frac{\bar{\alpha}_{\mathrm{s}} \sigma^{2}}{2} \bar{\delta}_{\mathrm{s}}-\bar{\alpha}_{\mathrm{s}} \operatorname{Cov}\left(X_{N}, \bar{Z}_{N}\right) \\
\hat{\alpha}_{\mathrm{s}}=\frac{1}{\sum_{i}\left(1 / \alpha_{\mathrm{s}, i}\right)}, \quad \delta_{\mathrm{s}}=\sum_{i} \delta_{\mathrm{s}, i}, \quad \hat{Z}_{N}=\sum_{i} Z_{N}^{(i)}
\end{gathered}
$$


By applying the equilibrium condition, $F_{\mathrm{S}}=F_{1}=\bar{F}$ and $\bar{\delta}_{\mathrm{s}}=\bar{\delta}_{1}=\bar{\delta}$, it holds that:

$$
\begin{gathered}
\frac{\bar{\alpha}_{\mathrm{s}} \sigma^{2}}{2} \bar{\delta}-\bar{\alpha}_{\mathrm{s}} \operatorname{Cov}\left(X_{N}, \bar{Z}_{N}\right)=-\frac{\bar{\alpha}_{1} \sigma^{2}}{2} \bar{\delta}-\bar{\alpha}_{1} \operatorname{Cov}\left(X_{N}, \bar{Y}_{N}\right) \\
\Leftrightarrow \frac{\left(\bar{\alpha}_{\mathrm{s}}+\bar{\alpha}_{1}\right) \sigma^{2}}{2} \bar{\delta}=\bar{\alpha}_{\mathrm{s}} \operatorname{Cov}\left(X_{N}, \bar{Z}_{N}\right)-\bar{\alpha}_{1} \operatorname{Cov}\left(X_{N}, \bar{Y}_{N}\right) \\
\Leftrightarrow \bar{\delta}=\frac{2\left\{\bar{\alpha}_{\mathrm{s}} \operatorname{Cov}\left(X_{N}, \bar{Z}_{N}\right)-\bar{\alpha}_{1} \operatorname{Cov}\left(X_{N}, \bar{Y}_{N}\right)\right\}}{\left(\bar{\alpha}_{\mathrm{s}}+\bar{\alpha}_{1}\right) \sigma^{2}}
\end{gathered}
$$

Finally, we obtain the following equilibrium future price:

$$
\begin{aligned}
\bar{F}-\mu & =\frac{\bar{\alpha}_{\mathrm{s}}\left\{\bar{\alpha}_{\mathrm{s}} \operatorname{Cov}\left(X_{N}, \bar{Z}_{N}\right)-\bar{\alpha}_{1} \operatorname{Cov}\left(X_{N}, \bar{Y}_{N}\right)\right\}}{\bar{\alpha}_{\mathrm{s}}+\bar{\alpha}_{1}}-\bar{\alpha}_{\mathrm{s}} \operatorname{Cov}\left(X_{N}, \bar{Z}_{N}\right) \\
& =-\frac{\bar{\alpha}_{\mathrm{s}} \bar{\alpha}_{1}}{\bar{\alpha}_{\mathrm{s}}+\bar{\alpha}_{\mathrm{s}}}\left\{\operatorname{Cov}\left(X_{N}, \bar{Y}_{N}\right)+\operatorname{Cov}\left(X_{N}, \bar{Z}_{N}\right)\right\} \\
& =-\bar{\alpha} \cdot \operatorname{Cov}\left(X_{N}, \bar{Y}_{N}+\bar{Z}_{N}\right), \quad \bar{\alpha}:=\frac{1}{1 / \bar{\alpha}_{\mathrm{s}}+1 / \bar{\alpha}_{\mathrm{s}}}
\end{aligned}
$$

\section{APPENDIX B COMPARISON WITH CARA-NORMAL MODEL}

Note that the equilibrium future price in (A.11) is closely related to that given by the so-called CARA-Normal equilibrium model (see Li (2004) and references therein), where "CARA" stands for constant absolute risk aversion and "Normal" indicates normal random variables. Let $\alpha_{i}$ be a risk aversion coefficient of an agent $i$ and $Y_{N}^{(k)}$ be a payoff from an asset $k \in[1, M]$ at time $N$. Define $\boldsymbol{y} \in \mathfrak{R}^{M}$ as the payoff vector whose $k$ th entry is given by $Y_{N}^{(k)}$ and $\boldsymbol{w}_{i} \in \mathfrak{R}^{M}$ as $\boldsymbol{w}_{i} \in \mathfrak{R}^{M}$ as the portfolio of agent $i$.

Consider the following problem.

PROBLEM 1 Assume that each agent solves the following optimization problem:

$$
\max _{\delta_{i}, \boldsymbol{w}_{i}} \mathbb{E}\left[\exp \left\{-\alpha_{i}\left(\left(\delta_{i}\left(X_{N}-F_{0}\right)+\boldsymbol{w}_{i}^{\top} \boldsymbol{y}\right)\right\}\right]\right.
$$

With this assumption, find an equilibrium price, where:

$$
\left[\begin{array}{c}
X_{N}-F_{0} \\
\boldsymbol{y}
\end{array}\right] \sim \mathcal{N}\left[\left[\begin{array}{c}
\mu-F_{0} \\
\boldsymbol{m}
\end{array}\right], \Theta\right], \quad \boldsymbol{m} \in \mathfrak{R}^{M}, \Theta \in \mathfrak{\Re}^{(M+1) \times(M+1)}
$$

The equilibrium price in Problem 1 may be derived as follows. First we compute the expectation in (B.1) as:

$$
\begin{aligned}
& \mathbb{E}\left[\operatorname { e x p } \left\{-\alpha_{i} l\left(\left(\delta_{i}\left(X_{N}-F_{0}+\boldsymbol{w}_{i}^{\top} \boldsymbol{y}\right)\right\}\right]\right.\right. \\
& \quad=\exp \left\{-\alpha_{i}\left[\delta_{i}, \boldsymbol{w}_{i}^{\top}\right]\left[\begin{array}{c}
\mu-F_{0} \\
\boldsymbol{m}
\end{array}\right]+\frac{\alpha_{i}^{2}}{2}\left[\delta_{i}, \boldsymbol{w}_{i}^{\top}\right] \Theta\left[\delta_{i} \boldsymbol{w}_{i}\right]\right\}
\end{aligned}
$$


By taking the first-order condition, we obtain:

$$
\begin{gathered}
-\alpha_{i}\left[\begin{array}{c}
\mu-F_{0} \\
\boldsymbol{m}
\end{array}\right]+\alpha_{i}^{2} \Theta\left[\begin{array}{c}
\delta_{i}^{*} \\
\boldsymbol{w}_{i}^{*}
\end{array}\right]=0 \\
\Leftrightarrow \Theta\left[\begin{array}{c}
\delta_{i}^{*} \\
\boldsymbol{w}_{i}^{*}
\end{array}\right]=\frac{1}{\alpha_{i}}\left[\begin{array}{c}
\mu-F_{0} \\
\boldsymbol{m}
\end{array}\right]
\end{gathered}
$$

We now compute the sum for both sides of Equation (B.4) over $i$ and apply an equilibrium condition:

$$
\sum_{i} \delta_{i}^{*}=0
$$

In this case:

$$
\Theta\left[\sum_{i}^{0} \boldsymbol{w}_{i}^{*}\right]=\sum_{i} \frac{1}{\alpha_{i}}\left[\begin{array}{c}
\mu-F_{0} \\
\boldsymbol{m}
\end{array}\right]
$$

holds. Let $\bar{\alpha}$ and $\overline{\boldsymbol{w}}$ satisfy:

$$
\frac{1}{\bar{\alpha}}=\sum_{i} \frac{1}{\alpha_{i}}, \quad \overline{\boldsymbol{w}}=\sum_{i} \boldsymbol{w}_{i}^{*}
$$

Then the equilibrium price is computed as follows:

$$
F_{0}=\mu-\bar{\alpha} \cdot \operatorname{Cov}\left(X_{N}, \overline{\boldsymbol{w}}^{\top} \boldsymbol{y}\right)
$$

If we assume that the optimal portfolio is fixed for each agent, condition (B.8) is essentially the same as that given in (A.11).

\section{APPENDIX C PROOF OF PROPOSITION 1}

First, we solve condition (21) with respect to $F_{S}(t)$. From (21), it holds that

$$
\begin{aligned}
\exp \left(\alpha_{\mathrm{s}} \int_{t_{0}}^{t} \delta_{\mathrm{S}}(t) F_{\mathrm{S}}(t) \mathrm{d} t\right) & =\mathbb{E}\left[\exp \left(\alpha_{\mathrm{s}} \int_{t_{0}}^{t} \delta_{\mathrm{s}}(t) X(t) \mathrm{d} t\right)\right] \\
& =\exp \left(\alpha_{\mathrm{s}} \bar{Z}+\frac{\alpha_{\mathrm{s}}^{2}}{2} \operatorname{Var}(Z)\right)
\end{aligned}
$$

where:

$$
Z:=\int_{t_{0}}^{t} \delta_{\mathrm{S}}(t) X(t) \mathrm{d} t, \quad \bar{Z}:=\mathbb{E}(Z)=\int_{t_{0}}^{t} \delta_{\mathrm{S}}(t) \mu(t) \mathrm{d} t
$$


Here $\operatorname{Var}(Z)$ can be computed as follows:

$$
\begin{aligned}
\operatorname{Var}(Z) & =\mathbb{E}\left[\left(\int_{t_{0}}^{t} \delta_{\mathrm{S}}(t)(X(t)-\mu(t)) \mathrm{d} t\right)^{2}\right] \\
& =\mathbb{E}\left[\left(\int_{t_{0}}^{t} \delta_{\mathrm{S}}(u)(X(u)-\mu(u)) \mathrm{d} u\right)\left(\int_{t_{0}}^{t} \delta_{\mathrm{S}}(v)(X(v)-\mu(v)) \mathrm{d} v\right)\right] \\
= & \mathbb{E}\left[\int_{t_{0}}^{t} \int_{t_{0}}^{t} \delta_{\mathrm{S}}(u) \delta_{\mathrm{S}}(v)(X(u)-\mu(u))(X(v)-\mu(v)) \mathrm{d} v \mathrm{~d} u\right] \\
= & \int_{t_{0}}^{t} \int_{t_{0}}^{t} \delta_{\mathrm{S}}(u) \delta_{\mathrm{S}}(v) \sigma(u) \sigma(v) \rho(u, v) \mathrm{d} v \mathrm{~d} u \\
& \rho(u, v):=\operatorname{Corr}(X(u), X(v)) \\
= & 2 \int_{t_{0}}^{t} \int_{t_{0}}^{u} \delta_{\mathrm{S}}(u) \delta_{\mathrm{S}}(v) \sigma(u) \sigma(v) \rho(u, v) \mathrm{d} v \mathrm{~d} u
\end{aligned}
$$

By substituting the above $\operatorname{Var}(Z)$ to $(C .1)$, we obtain

$$
\begin{aligned}
& \exp \left(\alpha_{\mathrm{S}} \int_{t_{0}}^{t} \delta_{\mathrm{S}}(u) F_{S}(u) \mathrm{d} t\right) \\
& =\exp \left(\alpha_{\mathrm{S}} \int_{t_{0}}^{t} \delta_{\mathrm{S}}(u) \mu(u) \mathrm{d} u+\alpha_{\mathrm{S}}^{2} \int_{t_{0}}^{t} \int_{t_{0}}^{u} \delta_{\mathrm{S}}(u) \delta_{\mathrm{S}}(v) \sigma(u) \sigma(v) \rho(u, v) \mathrm{d} v \mathrm{~d} u\right) \\
& \quad \Leftrightarrow \int_{t_{0}}^{t} \delta_{\mathrm{S}}(u) F_{S}(u) \mathrm{d} u \\
& =\int_{t_{0}}^{t} \delta_{\mathrm{S}}(u) \mu(u) \mathrm{d} u+\alpha_{\mathrm{S}} \int_{t_{0}}^{t} \delta_{\mathrm{S}}(u) \sigma(u) \int_{t_{0}}^{u} \delta_{\mathrm{S}}(v) \sigma(v) \rho(u, v) \mathrm{d} v \mathrm{~d} u
\end{aligned}
$$

If we differentiate both sides of Equation (C.3) with respect to $t$, we finally obtain the following:

$$
\begin{aligned}
& \delta(t) F_{S}(t)=\delta(t) \mu(t)+\alpha_{\mathrm{S}} \delta(t) \sigma(t) \int_{t_{0}}^{t} \delta(v) \sigma(v) \rho(t, v) \mathrm{d} v \\
& \Leftrightarrow F_{S}(t)=\mu(t)+\alpha_{\mathrm{S}} \int_{t_{0}}^{t} \delta(v) \sigma(t) \sigma(v) \rho(t, v) \mathrm{d} v
\end{aligned}
$$

Similarly, we solve (22) with respect to $F_{l}(t)$. First, it holds that:

$$
\begin{aligned}
(22) \Leftrightarrow & \exp \left(\alpha_{1} \int_{t_{0}}^{t} \delta_{1}(t) F_{l}(t) \mathrm{d} t\right) \mathbb{E}\left[\exp \left\{-\alpha_{1}\left(\int_{t_{0}}^{t}\left[\delta_{1}(t) X(t)+Y(t)\right] \mathrm{d} t\right)\right\}\right] \\
& =\mathbb{E}\left[\exp \left(-\alpha_{1} \int_{t_{0}}^{t} Y(t) \mathrm{d} t\right)\right]
\end{aligned}
$$


Let $M(t):=\delta_{1}(t) X(t)+Y(t)$, and compute the variance of $\int_{t_{0}}^{t} M(t) \mathrm{d} t$ as:

$$
\begin{aligned}
\mathbb{E} & {\left[\left(\int_{t_{0}}^{t}(M(t)-\bar{M}(t)) \mathrm{d} t\right)^{2}\right] } \\
& =\mathbb{E}\left[\left(\int_{t_{0}}^{t}(M(u)-\bar{M}(u)) \mathrm{d} u\right)\left(\int_{t_{0}}^{t}(M(v)-\bar{M}(v)) \mathrm{d} v\right)\right] \\
& =\mathbb{E}\left[\int_{t_{0}}^{t} \int_{t_{0}}^{t}(M(u)-\bar{M}(u))(M(v)-\bar{M}(v)) \mathrm{d} v \mathrm{~d} u\right] \\
& =\int_{t_{0}}^{t} \int_{t_{0}}^{t} \operatorname{Cov}(M(u), M(v)) \mathrm{d} v \mathrm{~d} u \\
& =2 \int_{t_{0}}^{t} \int_{t_{0}}^{u} \operatorname{Cov}(M(u), M(v)) \mathrm{d} v \mathrm{~d} u
\end{aligned}
$$

Then we have:

$$
\begin{aligned}
& \mathbb{E}\left[\exp \left\{-\alpha_{1}\left(\int_{t_{0}}^{t}\left[\delta_{1}(t) X(t)+Y(t)\right] \mathrm{d} t\right)\right\}\right] \\
& =\exp \left\{-\alpha_{1}\left(\int_{t_{0}}^{t}\left[\delta_{1}(u) \mu(u)+\bar{Y}(u)\right] \mathrm{d} u\right)\right. \\
& \left.\quad+\alpha_{1}^{2}\left(\int_{t_{0}}^{t} \int_{t_{0}}^{u} \operatorname{Cov}(M(u), M(v)) \mathrm{d} v \mathrm{~d} u\right)\right\} \\
& \mathbb{E}\left[\exp \left\{-\alpha_{1}\left(\int_{t_{0}}^{t} Y(t) \mathrm{d} t\right)\right\}\right] \\
& \quad=\exp \left\{-\alpha_{1}\left(\int_{t_{0}}^{t} \bar{Y}(u) \mathrm{d} u\right)+\alpha_{1}^{2}\left(\int_{t_{0}}^{t} \int_{t_{0}}^{u} \operatorname{Cov}(Y(u), Y(v)) \mathrm{d} v \mathrm{~d} u\right)\right\}
\end{aligned}
$$

By substituting (C.8) and (C.9) to (C.5), we find:

$$
\begin{aligned}
& \alpha_{1} \int_{t_{0}}^{t} \delta_{1}(u) F_{l}(u) \mathrm{d} u-\alpha_{1} \int_{t_{0}}^{t}\left[\delta_{1}(u) \mu(u)+\bar{Y}(u)\right] \mathrm{d} u \\
& \quad+\alpha_{1}^{2} \int_{t_{0}}^{t} \int_{t_{0}}^{u} \operatorname{Cov}(M(u), M(v)) \mathrm{d} v \mathrm{~d} u \\
& =-\alpha_{1} \int_{t_{0}}^{t} \bar{Y}(u) \mathrm{d} u+\alpha_{1}^{2} \int_{t_{0}}^{t} \int_{t_{0}}^{u} \operatorname{Cov}(Y(u), Y(v)) \mathrm{d} v \mathrm{~d} u
\end{aligned}
$$


where:

$$
\begin{aligned}
\operatorname{Cov} & M(u), M(v)) \\
= & \delta(u) \delta(v) \operatorname{Cov}(X(u), X(v))+\delta(u) \operatorname{Cov}(X(u), Y(v)) \\
& +\delta(v) \operatorname{Cov}(X(v), Y(u))+\operatorname{Cov}(Y(u), Y(v)) \\
= & \delta(u) \delta(v) \sigma(u) \sigma(v) \rho(u, v)+\delta(u) \sigma(u) \sigma_{y}(v) \operatorname{Corr}(X(u), Y(v)) \\
& +\delta(v) \sigma(v) \sigma_{y}(u) \operatorname{Corr}(X(v), Y(u))+\sigma_{y}(u) \sigma_{y}(v) \operatorname{Corr}(Y(u), Y(v))
\end{aligned}
$$

If we differentiate both sides of (C.10) with respect to $t$, we obtain:

$$
\begin{aligned}
\delta(t) F_{l}(t)= & \delta(t) \mu(t) \\
& -\alpha_{1} \int_{t_{0}}^{t}\left\{\delta(t) \delta(v) \sigma(t) \sigma(v) \rho(t, v)+\delta(t) \sigma(t) \sigma_{y}(v) \operatorname{Corr}(X(t), Y(v))\right. \\
& \left.+\delta(v) \sigma(v) \sigma_{y}(t) \operatorname{Corr}(X(v), Y(t))\right\} \mathrm{d} v
\end{aligned}
$$

Consequently:

$$
\begin{aligned}
F_{l}(t)= & \mu(t)-\alpha_{1} \int_{t_{0}}^{t}\{\delta(v) \sigma(t) \sigma(v) \rho(t, v) \\
& \left.+\sigma(t) \sigma_{y}(v) \operatorname{Corr}(X(t), Y(v))+\frac{\delta(v)}{\delta(t)} \sigma(v) \sigma_{y}(t) \operatorname{Corr}(X(v), Y(t))\right\} \mathrm{d} v
\end{aligned}
$$

holds.

\section{REFERENCES}

Cao, M., and Wei, J. (2004). Weather derivatives valuation and market price of weather risk. Journal of Futures Markets 24(11), 1065-1089.

Davis, M. (1997). Option pricing in incomplete markets. Mathematics of Derivative Securities, Dempster, M. A. H. and Pliska, S. R. (eds). Cambridge University Press, Cambridge.

Davis, M. (2001). Pricing weather derivatives by marginal value. Quantitative Finance 1, 305-308.

Cont, R., and Tankov, P. (2004). Financial Modeling With Jump Processes. Chapman \& $\mathrm{Hall} / \mathrm{CRC}$.

Geman, H. (1999). Insurance and Weather Derivatives. Risk Books, London.

Hastie, T., and Tibshirani, R. (1990). Generalized Additive Models. Chapman \& Hall, London.

Henderson, V., and Hobson, D. (2007). Utility indifference pricing-an overview. Indifference Pricing, Carmona, R. (ed). Princeton University Press, Princeton, NJ.

Kariya, T. (2003). Weather risk swap valuation. Working Paper, Institute of Economic Research, Kyoto University, Japan.

Journal of Risk 
Li, Q. (2004). Essays on asset pricing, consumption and wealth. PhD Dissertation, Stanford University. http://www.stanford.edu/japrimbs/ThesisQi.pdf.

Platen, E., and West, J. (2004). Fair pricing of weather derivatives. Asia-Pacific Financial Markets 11(1), 23-53. 